\title{
TENSÃO SUPERFICIAL DINÂMICA E ÂNGULO DE CONTATO DE SOLUÇÕES AQUOSAS COM SURFATANTES EM SUPERFÍCIES ARTIFICIAIS E NATURAIS
}

\author{
CRISTINA A. R. IOST ${ }^{1}$, CARLOS G. RAETANO ${ }^{2}$
}

RESUMO: O trabalho objetivou avaliar o efeito de surfatantes em soluções aquosas sobre a tensão superficial dinâmica e ângulo de contato das gotas em diferentes superfícies: artificiais (lâmina de vidro e de óxido de alumínio) e naturais (superfícies adaxiais de folhas de Euphorbia heterophylla, Ipomoea grandifolia e Brachiaria plantaginea). Seis formulações de surfatantes (Antideriva ${ }^{\circledR}$, Uno ${ }^{\circledR}$, Pronto $3^{\circledR}$, Li-700 ${ }^{\circledR}$, Supersil ${ }^{\circledR}$ e Silwet L-7 ${ }^{\circledR}$ ), respectivamente nas doses recomendadas do produto comercial $\left(0,050 ; 0,025 ; 0,100 ; 0,250 ; 0,100\right.$ e $\left.0,100 \% \mathrm{v} \mathrm{v}^{-1}\right)$ e o dobro delas, foram avaliadas em soluções aquosas. A tensão superficial dinâmica e o ângulo de contato formado sobre as superfícies naturais foram medidos por tensiômetro. Os ângulos de contato formados pelas gotas nas superfícies artificiais foram obtidos por análise de imagens capturadas por uma câmera digital. Os surfatantes influenciam nas propriedades físico-químicas de soluções aquosas. As soluções contendo os surfatantes Silwet L-77 ${ }^{\circledR}$ e Supersil ${ }^{\circledR}$, nas doses de 0,100 e $0,200 \%$ v v v ${ }^{-1}$, proporcionaram maiores reduções na tensão superficial dinâmica e menores ângulos de contato das gotas sobre as superfícies artificiais e naturais. Os surfatantes organossiliconados em solução aquosa foram mais eficientes na redução da tensão superficial e proporcionaram maior molhamento de superfícies natural e artificial. Em alvos naturais, essas propriedades obtidas com organossiliconados são dependentes das características de superfície das espécies vegetais.

PALAVRAS-CHAVE: adjuvantes, pulverização, propriedades físico-químicas, espécies vegetais.

\section{DYNAMIC SURFACE TENSION AND CONTACT ANGLE OF WATER SOLUTIONS WITH SPRAY SURFACTANTS IN ARTIFICIAL AND NATURAL SURFACES}

\begin{abstract}
The aim of the work was to evaluate the effect of surfactants in water solutions on dynamic surface tension and contact angle formed by the spray droplets in different surfaces: artificial (glass and aluminum oxide slides) and natural (leaves surface of three species of weeds: Euphorbia heterophylla, Ipomoea grandifolia e Brachiaria plantaginea). Were studied six surfactants formulations (Antideriva ${ }^{\mathrm{TM}}$, Uno ${ }^{\mathrm{TM}}$, Pronto $3^{\mathrm{TM}}, \mathrm{Li}^{-700^{\mathrm{TM}}}{ }^{\text {, Supersil }}{ }^{\mathrm{TM}}$ and Silwet L-7 ${ }^{\mathrm{TM}}$ ) in water solution using the dosage recommended by the manufacturer $(0.050 ; 0.025 ; 0.100 ; 0.250$; 0.100 and $0.100 \% \mathrm{v} \mathrm{v}^{-1}$ ) and twice that dose, respectively. The dynamic surface tension and the angle formed on the surfaces of the leaves were measured by tensiometer. The contact angles of the spray droplets in two surfaces (hydrophilic and hydrophobic) were obtained by the analysis of images captured by digital camera. This study reveals that the use of surfactants affect the physicalchemical properties of water solutions. The solutions with the surfactants Silwet L-77 ${ }^{\mathrm{TM}}$ and Supersil $^{\mathrm{TM}}$ for both appraised dosages provided the highest reductions in the dynamic surface tension and the smallest contact angles of the droplets on artificial and natural surfaces. The organosilicones surfactants in water solutions were more efficient in the reduction of the surface tension and increase the wetting area on artificial and natural surfaces. In natural targets these properties obtained with organosilicones are dependents of surface characteristics of the vegetal species.
\end{abstract}

KEYWORDS: adjuvant, spraying, physical-chemical properties, vegetal species.

\footnotetext{
${ }^{1}$ Mestre em Agronomia - Proteção de Plantas - Departamento de Produção Vegetal, FCA/UNESP, Câmpus de Botucatu - SP, crisiost@fca.unesp.br.

${ }^{2}$ Prof. Assistente Doutor, Departamento de Produção Vegetal, FCA/UNESP, Câmpus de Botucatu - SP.

Recebido pelo Conselho Editorial em: 25-8-2008

Aprovado pelo Conselho Editorial em: 27-3-2010
}

Eng. Agríc., Jaboticabal, v.30, n.4, p.670-680, jul./ago. 2010 


\section{INTRODUÇÃO}

No Brasil, há diversos produtos para uso agrícola que são recomendados para atuar junto à calda de pulverização com o intuito de modificar características físico-químicas das soluções. Estes produtos são denominados adjuvantes, os quais constituem um componente do produto formulado ou conferem características desejáveis à calda de pulverização, quando associados ao diluente, via de regra, a água. Os termos "adjuvantes" e "surfatantes" têm sido comumente usados ao se referir aos determinados grupos de substâncias sem defini-los adequadamente (DURIGAN, 1993; KISSMANN, 1997).

Os surfatantes são tidos como adjuvantes que atuam modificando as forças interfaciais, com orientação das suas moléculas entre as interfaces, promovendo ajustamento mais íntimo de duas substâncias (DURIGAN, 1993), ou, ainda, entre substância e superfície.

Desde 1973, tem-se o conhecimento do potencial de organossiliconados atuarem como surfatantes junto aos produtos fitossanitários, especialmente associados aos herbicidas. Entretanto, somente em 1985 foi comercializado o primeiro surfatante organossiliconado, denominado Silwet L-77 ${ }^{\circledR}$ (FOY, 1996).

Ainda não está claramente estabelecida a real necessidade de uso destes adjuvantes em pulverizações. Em muitos casos, a recomendação vai além das características da calda que estes produtos costumam modificar, necessitando de uma avaliação do alvo da pulverização, das condições edafoclimáticas e dos equipamentos utilizados na aplicação. Assim, estudos detalhados sobre a ação desses produtos na melhoria da eficácia biológica, manutenção da eficácia sob condições adversas, redução das perdas e contaminação ambiental são indispensáveis quando se busca maior retorno econômico (COMBELLACK, 1995).

Os estudos de tensão superficial estática e dinâmica são complementares. Para a determinação da tensão superficial estática, estabelece-se o tempo de formação das gotas e a concentração do surfatante capaz de atingir a tensão superficial mínima da solução (MENDONÇA et al., 2007). Ao contrário da tensão superficial dinâmica, que registra o tempo de duração de uma gota ou bolha de gás com elevado grau de pureza, a exemplo do nitrogênio, em outro fluido com concentração pre-estabelecida (tempo de interface). No entanto, pode-se determinar a tensão superficial dinâmica de soluções pelo método da gota pendente, utilizando-se de sistema de medidas dinâmicas com tensiômetro automático (IOST, 2008).

Pesquisas conduzidas por MONTÓRIO (2001), SUN \& FOY (1998) e por LO \& HOPKINSON (1995) revelaram que surfatantes organossiliconados, quando em soluções aquosas, promoveram menores valores de tensão superficial estática, chegando a $20 \mathrm{mN} \mathrm{m}^{-1}$. DEXTER \& HUDDLESTON (1998) estudaram a tensão superficial dinâmica de caldas de pulverização preparadas com o herbicida imazethapyr e adjuvantes em concentrações de 0,002 a $1,0 \% \mathrm{v} \mathrm{v}^{-1} \mathrm{e}$ constataram que concentrações superiores a $0,1 \% \mathrm{v} \mathrm{v}^{-1}$ da maioria dos adjuvantes em teste reduziram significativamente a tensão superficial dinâmica de soluções aquosas.

Segundo GREEN \& HAZEN (1998), a tensão superficial está relacionada entre as propriedades dos adjuvantes que influenciam na atividade biológica dos produtos fitossanitários. Com a redução da tensão superficial, têm-se os efeitos molhante (MENDONÇA et al., 1999), espalhante e penetrante (GREENE \& BUKOVAC, 1974) e maior velocidade de absorção e/ou translocação (MARTINS et. al., 2009).

Dessa forma, os adjuvantes que possuem a característica de modificar a tensão superficial são denominados surfatantes (DURIGAN, 1993; KISSMANN, 1997). No entanto, a redução da tensão superficial de soluções aquosas pode apresentar efeito antagônico pela adição de surfatantes aos produtos fitossanitários (MENDONÇA et al., 1999; COSTA et al., 2005; SILVA et al., 2006).

As gotas formadas durante o processo de pulverização, ao se depositarem nas plantas, formam ângulo de contato com a superfície. Ao medir o ângulo de contato com que uma gota fica na 
superfície, pode caracterizá-la quanto à capacidade de molhamento; quando menor que $90^{\circ}$, pode-se considerar que a superfície é molhada pelo líquido (hidrofílica), porém se os ângulos forem maiores que $90^{\circ}$, caracterizam-se superfícies hidrofóbicas (MOITA NETO, 2006). No caso da água, se a superfície de deposição for hidrófoba (com ceras), o contato será menor, a gota será esférica e o ângulo de contato será maior. Entretanto, se a superfície for mais hidrófila, a gota se espalhará, podendo até formar um filme uniforme. A molhabilidade das folhas das plantas depende dos constituintes de sua epiderme, onde a atração pela água precisa ser maior que a tensão superficial desse líquido para uma boa molhabilidade (KISSMANN, 1997; LO \& HOPKINSON, 1995).

O objetivo do trabalho foi avaliar o efeito de adjuvantes sobre a tensão superficial dinâmica e o ângulo de contato de soluções aquosas em superfícies artificiais e naturais.

\section{MATERIAL E MÉTODOS}

\section{Tensão superficial dinâmica e ângulo de contato das gotas em alvo natural}

As avaliações de tensão superficial dinâmica e ângulo de contato nas superfícies vegetais foram realizadas no Laboratório de Físico-Química de Superfícies do Departamento de Química da Faculdade de Filosofia, Ciências e Letras FFCL/USP, Câmpus de Ribeirão Preto - SP, nos dias 11 e 13 de setembro de 2007. Para a avaliação, foram preparadas soluções contendo água destilada e os surfatantes listados nas Tabelas 1 e 2 , nas concentrações: $5 ; 10 ; 20 ; 40 ; 50 ; 100$ e 200\%, das doses recomendadas para cada produto comercial.

O equipamento utilizado para a determinação da tensão superficial dinâmica foi um tensiômetro automático, modelo OCA-20, do fabricante Dataphysics Germany (sistema de medidas dinâmicas), em que a tensão das soluções foi determinada pelo método da gota pendente. Uma cubeta de vidro óptico contendo água foi utilizada para conter a evaporação da gota. A imagem da gota das soluções, formada em seringa até o termostato óptico, foi capturada usando uma câmera CCD, que através da captura da imagem analisa o formato da gota por assimetria de eixos (ADSA axisymmetric drop shape analysis).

Um software específico que utiliza uma posição ideal como linha de referência no campo de imagem possibilitou identificar o ponto-chave para o início da gravação das imagens, até mesmo antes da formação completa da gota. A tensão superficial foi determinada pela digitalização e análise do perfil da gota, utilizando para ajuste a eq.(1) de Young-Laplace, a qual foi considerada a tensão superficial de equilíbrio somente após alcançar valor constante por, pelo menos, dois minutos.

em que,

$$
\mathrm{Pi}-\mathrm{Pe}=(\mathrm{D}-1) \sigma / \mathrm{R}
$$

$\mathrm{Pi}$ - pressão interna, $\mathrm{Pa}$;

$\mathrm{Pe}$ - pressão externa, $\mathrm{Pa}$;

$\sigma$ - tensão superficial, $\mathrm{N}$;

$\mathrm{R}$ - raio da gota, $\mathrm{m}$, e

D - dimensão espacial, $\mathrm{m}$.

Para a avaliação do ângulo de contato das gotas nas superfícies foliares, foi utilizado o mesmo equipamento, pelo qual também se obtém o valor de $\theta$ pela análise da imagem. Os ângulos dos lados direito e esquerdo da imagem da gota formada com as diferentes soluções foram calculados e automaticamente estabelecido o $\theta$ médio, o qual foi denominado ângulo de contato de equilíbrio depois de alcançar um valor constante por, pelo menos, dois minutos. Como padrão de comparação, foi calculado também o ângulo formado nas superfícies pela água destilada. 
TABELA 1. Características dos adjuvantes utilizados como tratamentos em soluções aquosas. Characteristics of the adjuvant used in water solutions.

\begin{tabular}{|c|c|c|c|}
\hline $\begin{array}{c}\text { Produto } \\
\text { Comercial } \\
\end{array}$ & Ingrediente Ativo & Grupo Químico & Classificação \\
\hline Antideriva ${ }^{\circledR}$ & nonil fenol etoxilado & etoxilado & tensoativo/emulsionante \\
\hline Uno ${ }^{\circledR}$ & nonil fenol etoxilado & etoxilado & tensoativo/antideriva \\
\hline Pronto $3{ }^{\circledR}$ & lecitina & lecitina & antideriva/antiespumante \\
\hline $\operatorname{Li} 700{ }^{\circledR}$ & lecitina e ácido propiônico & $\begin{array}{l}\text { lecitina e ácido } \\
\text { propiônico }\end{array}$ & surfatante/acidificador \\
\hline Supersol ${ }^{\circledR}$ & polímero glicol silano & organossiliconado & espalhante adesivo \\
\hline Silwet L-77 ${ }^{(B)}$ & copolímero de poliéter e silicone & organossiliconado & agente molhante \\
\hline
\end{tabular}

TABELA 2. Doses dos adjuvantes utilizadas para a determinação da tensão superficial dinâmica de equilíbrio das soluções. Dosages of the adjuvant used to determination of the equilibrium dynamic surface tension of the solutions.

\begin{tabular}{ccccccc}
\hline $\begin{array}{c}\% \text { da Dose } \\
\text { Recomendada }\end{array}$ & \multicolumn{7}{c}{ Doses $\left(\% \mathrm{v} \mathrm{v}^{-1}\right)$} \\
\cline { 2 - 6 } & Antideriva $^{\circledR}$ & Uno $^{\circledR}$ & Pronto 3 $^{\circledR}$ & Li-700 $^{\circledR}$ & Supersil $^{\circledR}$ & Silwet L-77 $^{\circledR}$ \\
\hline 5 & 0,0025 & 0,00125 & 0,005 & 0,0125 & 0,005 & 0,005 \\
10 & 0,005 & 0,0025 & 0,010 & 0,0250 & 0,010 & 0,010 \\
20 & 0,010 & 0,0050 & 0,020 & 0,0500 & 0,020 & 0,020 \\
40 & 0,020 & 0,0100 & 0,040 & 0,1000 & 0,040 & 0,040 \\
50 & 0,025 & 0,0125 & 0,050 & 0,1250 & 0,050 & 0,050 \\
100 & 0,050 & 0,0250 & 0,100 & 0,2500 & 0,100 & 0,100 \\
200 & 0,100 & 0,0500 & 0,200 & 0,5000 & 0,200 & 0,200 \\
\hline
\end{tabular}

Observação: $\mathrm{v} \mathrm{v}^{-1}=$ volume por volume

As avaliações do ângulo de contato na superfície das folhas foram realizadas apenas com as concentrações de 100 e 200\% da dose recomendada dos adjuvantes (Tabela 3). Dois litros de solução foram preparados 10 minutos antes dos testes, utilizando-se de água destilada como solvente.

TABELA 3. Doses recomendadas dos adjuvantes e o dobro delas em solução aquosa para avaliação do ângulo das gotas. Dosages recommended of the adjuvants and the twice dosages in water solution to evaluation of the droplets angle.

\begin{tabular}{ccc}
\hline & \multicolumn{2}{c}{ Dosagens $\left(\% \mathrm{v} \mathrm{v}^{-1}\right)$} \\
\cline { 2 - 3 } Produto & Recomendada & Dobro \\
\hline Antideriva $^{\circledR}$ & 0,050 & 0,100 \\
Uno $^{\circledR}$ & 0,025 & 0,050 \\
Pronto 3 $^{\circledR}$ & 0,100 & 0,200 \\
Li $700^{\circledR}$ & 0,250 & 0,500 \\
Supersil $^{\circledR}$ & 0,100 & 0,200 \\
Silwet L-77 & & 0,200 \\
\hline
\end{tabular}

As folhas das espécies Euphorbia heterophylla (amendoim-bravo), Ipomoea grandifolia (corda-de-viola) e Brachiaria plantaginea (capim-marmelada) foram retiradas de vasos conduzidos em casa-de-vegetação. As folhas estudadas foram presas em lâmina de vidro com o auxílio de fita dupla face visando a evitar a rugosidade do limbo foliar.

Ao utilizar o dobro da dose dos adjuvantes, o maior molhamento da superfície foliar até a proximidade da nervura central, para alguns tratamentos, influenciou no ângulo médio de contato 
( $\theta$ ) superestimando-o. Essas leituras não foram descartadas, pois buscou realizar avaliações que se aproximassem ao máximo do que pode ocorrer a campo, dependendo da relação líquido- superfície.

As tensões superficiais dinâmicas, nas respectivas doses dos adjuvantes, foram comparadas pela forma gráfica em software específico, o qual possibilita a comparação múltipla da tendência das curvas de redução da tensão superficial de diferentes soluções.

\section{Ângulo de contato das gotas em alvo artificial}

As avaliações do ângulo de contato das gotas em superfícies artificiais $(\theta)$ foram realizadas no Laboratório do Grupo de Polímeros do Instituto de Física de São Carlos, IFSC/USP, no dia 06 de agosto de 2007. O volume das gotas formadas e estudadas para os respectivos tratamentos descritos na Tabela 1 foi de $3 \mu \mathrm{L}$ (microlitros), utilizando-se de seringa graduada com capacidade de $10 \mu \mathrm{L}$. Para estas avaliações, foram selecionados dois tipos de superfícies, uma com características hidrofóbicas, nesse caso, representada por lâmina de óxido de alumínio ( $\theta$ água $>90^{\circ}$ ), e outra com características hidrofílicas ( $\theta$ água $<90^{\circ}$ ), constituída por uma lâmina de vidro. Entre uma avaliação e a subsequente às lâminas, foram devidamente lavadas com água e secas com o auxílio de um jato de nitrogênio para que não ficasse nenhuma partícula que pudesse comprometer as demais avaliações.

O sistema utilizado para as avaliações foi composto por uma câmera fotográfica digital CCD, marca LG, posicionada à frente de uma plataforma, onde foram posicionadas as lâminas para avaliação do ângulo de contato das gotas formadas por uma seringa de $10 \mu \mathrm{L}$, suspensa acima e muito próxima da lâmina, com espaço suficiente para a formação das gotas de $3 \mu \mathrm{L}$. À frente do suporte das lâminas, foi posicionada uma lâmpada de filamento único, funcionando como um "flash" para garantir a nitidez da imagem. O comando para captura da imagem foi realizado pelo software Image Pro Express, onde, após a captura da imagem, a mesma foi salva e analisada por um segundo programa denominado Surftens. Para cada tratamento, foram utilizadas quatro gotas, as quais foram representadas pelas repetições.

A dificuldade durante esta avaliação foi registrar o momento exato em que a gota formada cessava a movimentação; instante em que a gota permanecia sem modificar o formato, para que a imagem da gota depositada sobre a superfície fosse captada. Os dados foram submetidos à análise de variância pelo teste $\mathrm{F}$, e as médias, comparadas pelo teste de Tukey, a 5\% de probabilidade. $\mathrm{O}$ programa estatístico utilizado foi o Sisvar 5.0 (FERREIRA, 2000).

\section{RESULTADOS E DISCUSSÃO}

Os surfatantes organossiliconados Silwet $\mathrm{L}-77^{\circledR}$ e Supersil ${ }^{\circledR}$ proporcionaram os menores valores de tensão superficial dinâmica, de forma similar aos resultados de tensão superficial estática com organossiliconados obtidos por MONTÓRIO (2001) e por SUN \& FOY (1998) (Tabela 4 e Figura 1). Para Silwet L-77 ${ }^{\circledR}$, mesmo a $5 \%$ da dose recomendada, foi possível observar redução significativa da tensão superficial dinâmica da solução. Os surfatantes Li-700 ${ }^{\circledR}$, Antideriva ${ }^{\circledR}$ e Uno ${ }^{\circledR}$ apresentaram comportamento semelhante na redução da tensão superficial, especialmente os dois últimos pertencentes ao mesmo grupo químico, com valores de tensão superficial mínima próximos de $30 \mathrm{mN} \mathrm{m}^{-1}$.

O surfatante Pronto $3^{\circledR}$ apresentou menor poder de redução da tensão superficial, comparativamente aos demais surfatantes em teste, com valor de tensão superficial dinâmica mínima de $45,11 \mathrm{mN} \mathrm{m}^{-1}$. Outros estudos também evidenciaram que os organossiliconados são os que possuem a maior capacidade de redução da tensão superficial da água, em relação aos demais grupos de adjuvantes, chegando a valores próximos a $20 \mathrm{mN} \mathrm{m}^{-1}$ (DEXTER \& HUDDLESTON, 1998; SUN \& FOY, 1998; MONTÓRIO, 2001). No entanto, MENDONÇA et al. (1999) e COSTA et al. (2005) constataram efeito antagônico na redução da tensão superficial estática ao adicionar o surfatante Silwet L- $77^{\circledR}(0,05 \%)$ ao herbicida glyphosate em solução aquosa. 
SILVA et al. (2006) também evidenciaram efeito antagônico nessa propriedade com a adição do surfatante etoxilado Aterbane $\mathrm{BR}^{\circledR}(0,025 \%)$ ao íon Fe (10 e 100 ppm).

TABELA 4. Valores da tensão superficial dinâmica de adjuvantes em diferentes concentrações (\% em relação à dose recomendada) em soluções aquosas. Values of the dynamic surface tension of adjuvant in different concentrations (\% in relation to the dosage recommended) in water solutions.

\begin{tabular}{|c|c|c|c|c|c|c|}
\hline \multirow{2}{*}{$\begin{array}{c}\% \text { da } \\
\text { Concentração }\end{array}$} & \multicolumn{6}{|c|}{ Tensão Superficial $\left(\mathrm{mN} \mathrm{m}^{-1}\right)$} \\
\hline & Antideriva $\left.^{(}\right)$ & Uno $^{\circledR}$ & Pronto $3^{\circledR}$ & Supersil $^{\circledR}$ & $\mathrm{Li}-700^{\circledR}$ & SilwetL-77 ${ }^{\circledR}$ \\
\hline $5 \%$ & 45,47 & 43,46 & 72,71 & 27,11 & 45,32 & 21,00 \\
\hline $10 \%$ & 43,23 & 41,34 & 72,66 & 24,68 & 40,35 & 20,11 \\
\hline $20 \%$ & 36,59 & 38,15 & 54,52 & 23,97 & 33,12 & 19,49 \\
\hline $40 \%$ & 33,26 & 31,88 & 51,26 & 21,99 & 32,50 & 19,45 \\
\hline $50 \%$ & 31,17 & 31,84 & 48,51 & 21,07 & 32,78 & 19,46 \\
\hline $100 \%$ & 31,81 & 30,60 & 44,77 & 21,23 & 32,90 & 19,43 \\
\hline $200 \%$ & 31,21 & 30,53 & 45,11 & 21,09 & 32,98 & 19,40 \\
\hline
\end{tabular}

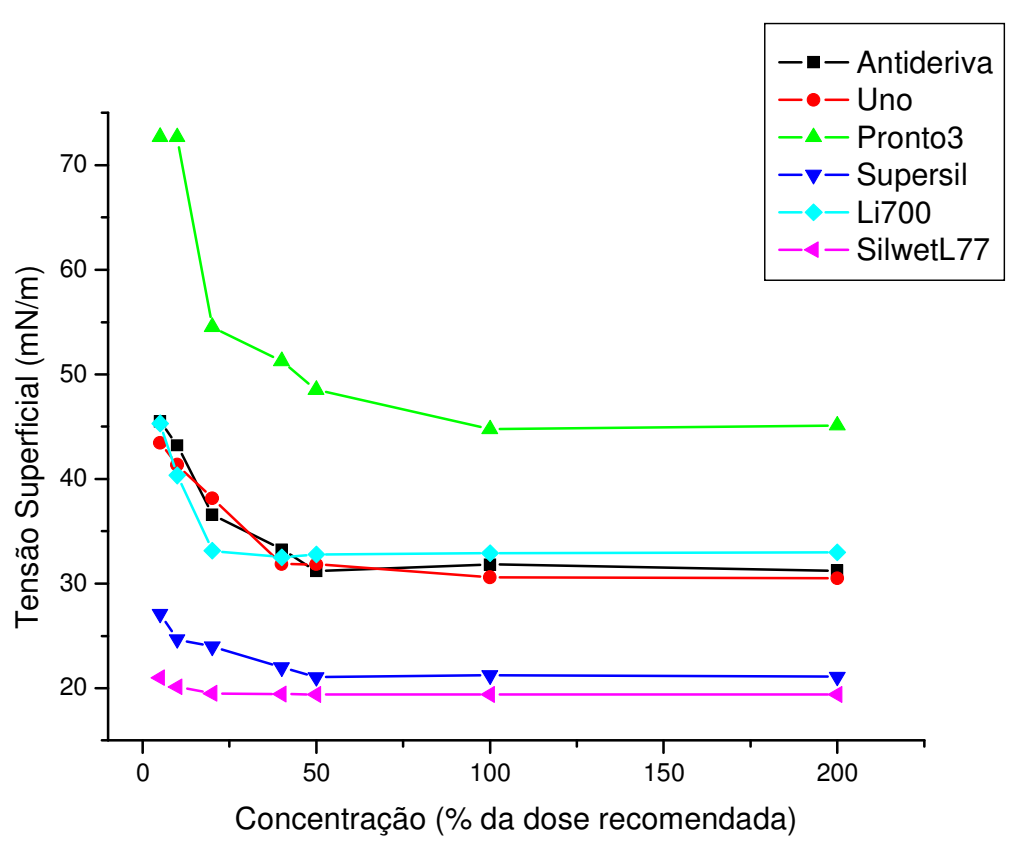

FIGURA 1. Tensão superficial dinâmica de soluções aquosas, em função da concentração de adjuvante, determinada pelo método da gota pendente em tensiômetro automático. Dynamic surface tension of water solutions, in function of the adjuvant concentration, established by pendent droplet method in automatic tensiometer.

Os surfatantes, quando adicionados à água, formam arranjos de maneira que a extremidade polar dessas moléculas fique voltada para a água, e a outra extremidade voltada para a pra atmosfera ou para a interface em que o líquido está em contato. Este arranjo das moléculas é chamado de micelas (DURIGAN, 1993). A formação de micelas, contudo, não ocorre em qualquer concentração, apenas a partir de uma concentração mínima, denominada de Concentração Micelar Crítica (CMC). Cada surfatante possui uma CMC, sendo possível identificá-la pela medição da tensão superficial da solução.

Dessa forma, a partir de uma concentração do surfatante na solução em que o valor da tensão não tiver mais alterações significativas, considera-se atingida a CMC a partir daquele ponto (DEXTER \& HUDDLESTON, 1998). No caso do Silwet L-77 ${ }^{\circledR}$ e Antideriva ${ }^{\circledR}$, a CMC foi, respectivamente, entre 5 e $10 \%$ e 40 a $50 \%$ da dose recomendada. Para Supersil ${ }^{\circledR}$ e Li-700 ${ }^{\circledR}$, esta concentração foi entre 20 e $40 \%$ da dose recomendada, ao contrário de Uno ${ }^{\circledR}$ e Pronto $3^{\circledR}$, onde a CMC foi compreendida entre 50 e $100 \%$ da dose recomendada. 
Quando comparadas as características das superfícies foliares, verificou-se que as folhas de amendoim-bravo são mais hidrofóbicas $\left(\theta\right.$ da água $\left.>90^{\circ}\right)$ em comparação às demais espécies vegetais (Tabela 5), conforme método para estudo de molhabilidade em superfícies proposto por MOITA NETO (2006). MENDONÇA (2003), ao avaliar as superfícies foliares de espécies vegetais, observou que as folhas de Euphorbia heterophylla (amendoim-bravo) possuem numerosos cristais de ceras na epicuticula.

Já as células epidérmicas da superfície adaxial de corda-de-viola (Ipomoea grandifolia) apresentam cera epicuticular em forma de estrias paralelas quando localizadas ao redor da célula epidérmica e entrelaçadas como uma rede no centro da célula. Isto significa que não apenas a presença dos cristais de cera, mas também o formato em que eles se apresentam pode ter influência sobre a molhabilidade da folha.

Durante a avaliação dos ângulos de contato das soluções com a superfície, também foi possível observar presença de tricomas nas folhas de amendoim-bravo. Portanto, o ângulo de contato formado depende não apenas da solução que está sendo aplicada, mas também das características da superfície, a exemplo de superfícies foliares que podem apresentar tricomas ou textura cerosa (KISSMANN, 1997).

Mais uma vez os surfatantes organossiliconados Silwet L- $77^{\circledR}$ e Supersil ${ }^{\circledR}$ apresentaram os $^{\circledR}$ menores ângulos de contato formados pelas gotas com a superfície (Tabela 5). Com relação ao produto Silwet L-77 ${ }^{\circledR}$, o fato de o ângulo de contato da gota ter sido maior para concentração de $200 \% \mathrm{v} \mathrm{v}^{-1}$ da dose recomendada, deve-se ao local da folha em que a gota foi depositada, próximo à nervura central.

Para as avaliações com o dobro da dose recomendada de Silwet L- $77^{\circledR}$ em capim-marmelada, a gota foi "barrada" pela nervura central da folha, o que provavelmente também aconteceu com as soluções contendo os surfatantes Li- $700^{\circledR}$ e Pronto $3^{\circledR}$, ambos na dose recomendada. Já para o amendoim-bravo, tricomas presentes na superfície foliar impediram o espalhamento de um dos lados da gota.

TABELA 5. Valores de ângulo de contato $(\theta)$ formado por gotas de soluções aquosas com adjuvantes em diferentes espécies de plantas daninhas. Values of contact angle ( $\boldsymbol{\theta})$ formed by the droplets of water solutions with adjuvant in different species of weeds.

\begin{tabular}{cccc}
\hline Tratamento & \multicolumn{3}{c}{ Valores de $\theta$} \\
\cline { 2 - 4 } & Corda-de-viola & Amendoim-bravo & Capim-marmelada \\
\hline Água & $78,4^{\circ}$ & $90,3^{\circ}$ & $60,9^{\circ}$ \\
Antideriva (100\%) & $43,3^{\circ}$ & $77,4^{\circ}$ & $30,4^{\circ}$ \\
Antideriva (200\%) & $39,0^{\circ}$ & $80,2^{\circ}$ & $21,4^{\circ}$ \\
Uno (100\%) & $63,2^{\circ}$ & $82,8^{\circ}$ & $77,0^{\circ}$ \\
Uno (200\%) & $45,5^{\circ}$ & $55,2^{\circ}$ & $53,2^{\circ}$ \\
Pronto 3 (100\%) & $53,3^{\circ}$ & $76,5^{\circ}$ & $88,2^{\circ}$ \\
Pronto 3 (200\%) & $66,2^{\circ}$ & $40,2^{\circ}$ & $78,4^{\circ}$ \\
LI-700 (100\%) & $68,0^{\circ}$ & $83,7^{\circ}$ & $80,0^{\circ}$ \\
LI-700 (200\%) & $64,7^{\circ}$ & $43,9^{\circ}$ & $57,8^{\circ}$ \\
Supersil (100\%) & $1,9^{\circ}$ & $4,1^{\circ}$ & $5,5^{\circ}$ \\
Supersil (200\%) & $0,0^{\circ}$ & $2,1^{\circ}$ & $0,0^{\circ}$ \\
Silwet L-77 (100\%) & $0,0^{\circ}$ & $5,6^{\circ}$ & $2,1^{\circ}$ \\
Silwet L-77 (200\%) & $0,0^{\circ}$ & $13,3^{\circ}$ & $11,0^{\circ}$ \\
\hline
\end{tabular}


Através dos resultados, observou-se relação inversa entre tensão superficial dinâmica e o ângulo de contato das gotas $(\theta)$, indicando que, quanto maior a tensão superficial, menor será a molhabilidade da superfície e, consequentemente, maior será o valor do ângulo de contato.

Dessa forma, o Pronto $3^{\circledR}$ apresentou os maiores valores de tensão superficial e, consequentemente, menor contribuição para a redução do ângulo de contato das gotas junto à superfície foliar das espécies vegetais, especialmente sobre o capim-marmelada.

Os ângulos formados sobre a lâmina de vidro foram menores que aqueles obtidos sobre a superfície de óxido de alumínio para a maioria dos produtos testados. Para as soluções contendo Silwet L-7 ${ }^{\circledR}$, os valores de $\theta$ foram iguais a $0^{\circ}$ para todas as concentrações em teste, tanto na superfície hidrofílica quanto na hidrofóbica (Tabelas 6 e 7). SUN \& FOY (1998), ao estudarem o ângulo de contato formado por soluções contendo surfatantes organossiliconados, em lâmina constituída por um filme uniforme, também constataram que este grupo de adjuvantes produziu gotas com ângulo igual a $0^{\circ}$, enquanto os demais adjuvantes (não siliconados) apresentaram valores de 56,2 a $86,5^{\circ}$.

Para a superfície de vidro, a quantidade dos surfatantes Li-700 ${ }^{\circledR}$, Pronto $3^{\circledR}$ e Uno ${ }^{\circledR}$ (dose recomendada e o dobro da dose), adicionada à solução aquosa, não foi significativa para alterar o ângulo de contato $(\theta)$ das gotas. Com exceção dos surfatantes siliconados (Silwet L-77 ${ }^{\circledR} \mathrm{e}$ Supersil ${ }^{\circledR}$ ) que apresentaram ângulo de contato igual a $0^{\circ}$, e do produto Antideriva ${ }^{\circledR}$, os demais surfatantes não apresentaram valores de $\theta$ significativamente diferentes dos encontrados para $o$ tratamento contendo apenas água (Tabela 6).

TABELA 6. Médias dos ângulos de contato $(\theta)$ obtidos por gotas formadas de soluções contendo adjuvantes e pela água sobre superfície de vidro (hidrofílica). Means of the contact angles $(\theta)$ obtained by droplets formed of solutions contained adjuvant and water on glass surface (hydrophilic).

\begin{tabular}{|c|c|c|}
\hline \multirow{2}{*}{ Adjuvante } & \multicolumn{2}{|c|}{ Valores Médios de $\theta\left(^{\circ}\right)$} \\
\hline & Dose & 2x Dose \\
\hline Antideriva & $15,00 \mathrm{~b}$ & $19,00 \mathrm{~b}$ \\
\hline Uno & $50,25 \mathrm{a}$ & $50,25 \mathrm{a}$ \\
\hline Pronto 3 & $60,50 \mathrm{a}$ & $61,75 \mathrm{a}$ \\
\hline $\mathrm{Li}-700$ & $49,25 \mathrm{a}$ & $49,25 \mathrm{a}$ \\
\hline Supersil & $0,00 \mathrm{~b}$ & $0,00 \mathrm{~b}$ \\
\hline Silwet L-77 & $0,00 \mathrm{~b}$ & $0,00 \mathrm{~b}$ \\
\hline Água & $57,50 \mathrm{a}$ & $57,50 \mathrm{a}$ \\
\hline $\mathrm{F}($ dose $)$ & \multicolumn{2}{|c|}{$0,13 \mathrm{~ns}$} \\
\hline F (trat.) & \multicolumn{2}{|c|}{$42,89 *$} \\
\hline C.V. $(\%)$ & \multicolumn{2}{|c|}{25,08} \\
\hline DMS & \multicolumn{2}{|c|}{19,85} \\
\hline
\end{tabular}

ns - não significativo a 5\% de probabilidade; * significativo a 5\% de probabilidade. Médias seguidas por mesma letra, minúscula na coluna, não diferem entre si, pelo teste de Tukey $(\mathrm{P}>0,05)$.

Na lâmina de óxido de alumínio, os ângulos de contato das gotas d'água foram os que apresentaram os maiores valores quando comparados aos demais ângulos das gotas contendo surfatantes Silwet L-77 ${ }^{\circledR}$ e Supersil ${ }^{\circledR}$, os quais apresentaram os menores valores médios. O aumento da dose não influenciou nos valores de ângulo de contato $(\theta)$ das soluções contendo os surfatantes Pronto $3^{\circledR}$, Li-700 ${ }^{\circledR}$, Supersil ${ }^{\circledR}$ e Silwet L-77 ${ }^{\circledR}$, sendo significativo apenas para Uno ${ }^{\circledR}$ e Antideriva ${ }^{\circledR}$, onde, com o dobro da dose, a molhabilidade das gotas sobre a superfície hidrofóbica foi maior (Tabela 7). 
TABELA 7. Médias dos ângulos de contato $(\theta)$ obtidos por gotas formadas de soluções contendo adjuvantes e água sobre superfície de alumínio oxidado (hidrofóbica). Means of the contact angles $(\theta)$ obtained by droplets formed of solutions contained adjuvant and water on aluminum oxide surface (hydrophobic).

\begin{tabular}{|c|c|c|}
\hline \multirow{2}{*}{ Adjuvante } & \multicolumn{2}{|c|}{ Valores médios de $\theta\left({ }^{\circ}\right)$} \\
\hline & Dose & 2x Dose \\
\hline Antideriva & 44,50 c $\mathrm{A}$ & $15,25 \mathrm{~d} \mathrm{~B}$ \\
\hline Uno & $66,25 \mathrm{~b} \mathrm{~A}$ & 56,25 b c B \\
\hline Pronto 3 & 71,25 b A & $70,00 \mathrm{~b} \mathrm{~A}$ \\
\hline $\mathrm{Li}-700$ & $59,00 \mathrm{~b}$ c A & $56,50 \mathrm{bc} \mathrm{A}$ \\
\hline Supersil & $8,25 \mathrm{~d} \mathrm{~A}$ & $2,50 \mathrm{~d} A$ \\
\hline Silwet L-77 & $0,00 \mathrm{~d} A$ & $0,00 \mathrm{~d} A$ \\
\hline Água & 95,75 a $\mathrm{A}$ & 95,75 a $\mathrm{A}$ \\
\hline $\mathrm{F}($ dose $)$ & \multicolumn{2}{|c|}{$20,38 *$} \\
\hline F (trat.) & \multicolumn{2}{|c|}{$113,11^{*}$} \\
\hline C.V. $(\%)$ & \multicolumn{2}{|c|}{14,62} \\
\hline DMS & \multicolumn{2}{|c|}{15,29} \\
\hline
\end{tabular}

* significativo a 5\% de probabilidade; médias seguidas por mesma letra, minúscula na coluna, não diferem entre si, pelo teste de Tukey $(\mathrm{P}>0,05)$; médias seguidas por mesma letra, maiúscula na linha, não diferem entre si, pelo teste de Tukey $(\mathrm{P}>0,05)$.

Apesar de o Silwet L- $77^{\circledR}$ ter sido mais efetivo em atingir a tensão superficial dinâmica de equilíbrio (valor constante por, pelo menos, dois minutos) e o ângulo de contato $(\theta)$ de zero grau, essas características não garantem o espalhamento da solução, uma vez que as superfícies das folhas das espécies vegetais irão determinar o comportamento dessa propriedade físico-química, conforme constatado para as espécies estudadas. Essa constatação foi registrada por LO \& HOPKINSON (1995), onde o Silwet L-77 ${ }^{\circledR}$ a $0,01 \%$ em solução aquosa reduziu a tensão superficial dinâmica da água a $20,9 \mathrm{mN} \mathrm{m}^{-1}$ e reduziu o ângulo de contato das gotas a zero grau. Entretanto, não sendo suficiente para molhar a superfície vegetal pilosa de Abutilon theophrasti, assim como a superfície artificial 3M-AF 4300 (filme para transparência). Os autores constataram que a concentração mínima do surfatante em água necessária para o molhamento espontâneo nessas superfícies foi de $0,05 \%$. Quando tais propriedades físico-químicas foram avaliadas em superfície do tipo Parafilm ${ }^{\circledR}$, a concentração necessária para propiciar o espalhamento da solução foi de 1,0\%.

\section{CONCLUSÕES}

O grupo químico e a dose dos surfatantes interferem nas propriedades físico-químicas de soluções aquosas. Os surfatantes siliconados Silwet L- $77^{\circledR}$ e Supersil ${ }^{\circledR}$, em solução aquosa, foram mais eficientes na redução da tensão superficial e proporcionaram maior molhamento de superfícies natural e artificial. Em alvos naturais, os maiores níveis de molhamento com soluções aquosas foram obtidos através das menores tensões superficiais e ângulos de contato das gotas, porém essa propriedade é dependente das características da superfície das espécies vegetais.

\section{REFERÊNCIAS}

COMBELLACK, H. Adjuvants-are they worthwhile? In: INTERNATIONAL SYMPOSIUM ON ADJUVANTS FOR AGROCHEMICALS, 14., 1995, Melbourne. Proceedings... Rotorua: New Zealand Forest Research Institute, 1995. p.336-344. (FRI Bulletin, 193).

COSTA, N.V.; MARTINS, D.; RODELLA, R.A.; COSTA, L.D.N.C. pH foliar e deposição de gotas de pulverização em plantas daninhas aquáticas: Brachiaria mutica, Brachiaria subquadripara e Panicum repens. Planta Daninha, Rio de Janeiro, v.23, n.2, p.295-304, 2005. 
DEXTER, R.W.; HUDDLESTON, E.W. Effects of adjuvants and dynamic surface tension on spray properties under simulated aerial conditions. In: NALEWAJA, J.D.; GROSS, G.R.; TANN, R.S. (Ed.) Pesticide formulations and application systems. West Conshohocken: American Society for Testing and Materials, 1998. v.18, p.95-105.

DURIGAN, J.C. Efeito de adjuvantes na aplicação e eficácia dos herbicidas. Jaboticabal: FUNEP, 1993. $42 \mathrm{p}$.

FERREIRA, D.F. Análises estatísticas por meio do Sisvar para Windows versão 4.0. In: REUNIÃO ANUAL DA REGIÃO BRASILEIRA DA SOCIEDADE INTERNACIONAL DE BIOMETRIA, 45., 2000, São Carlos. Anais... São Carlos: UFSCar, 2000. p.255-258.

FOY, C.L. Adjuvants: current trends and technology. In: FOY, C.L.; PRITCHARD, D.W. (Ed.) Pesticide formulation and adjuvant technology. Boca Raton: CRC Press LLC, 1996. chapter 15, p.323-352.

GREEN, J.M.; HAZEN, J.L. Understanding and using adjuvants properties to enhance pesticide activity. In: INTERNATIONAL SYMPOSIUM ON ADJUVANTS FOR AGROCHEMISTS, 5. 1998, Tennessee. Proceedings... Memphis: ISAA, 1998. p.25-36.

GREENE, D.W.; BUKOVAC, M. Stomatal penetration: effect of surfactants and role in foliar absorption. American Journal of Botany, Columbus, v.61, n.1, p.100-106, 1974.

IOST, C.A.R. Efeito de adjuvantes nas propriedades físico-químicas da água e na redução de deriva em pulverizações sobre diferentes espécies de plantas daninhas. 2008. 63 f. Dissertação (Mestrado em Proteção de Plantas) - Faculdade de Ciências Agronômicas, Universidade Estadual Paulista, Botucatu, 2008.

KISSMANN, K.G. Adjuvantes para caldas de produtos fitossanitário. In: CONGRESSO BRASILEIRO DA CIÊNCIA DAS PLANTAS DANINHAS, 21., 1997, Caxambu. Palestras... Caxambu: Sociedade Brasileira da Ciência das Plantas Daninhas, 1997. p.61-77.

LO, C.C.; HOPKINSON, M. Influence of adjuvants on droplet spreading. In: INTERNATIONAL SYMPOSIUM ON ADJUVANTS FOR AGROCHEMICALS, 14., 1995, Melbourne.

Proceedings... Rotorua: New Zealand Forest Research Institute, 1995. p.144-149. (FRI Bulletin, 193).

MARTINS, D.; CARBONARI, C.A.; TERRA, M.A.; MARCHI, S.R. Ação de adjuvantes na absorção e translocação de glyphosate em plantas de aguapé (Eichhornia crassipes). Planta Daninha, Rio de Janeiro, v.27, n.1, p.155-163, 2009.

MENDONÇA, C.G. Efeito de óleos minerais e vegetais nas propriedades físico-químicas das caldas de pulverização e suas interações com superfícies foliares. 2003. $96 \mathrm{f}$. Tese (Doutorado em Proteção de Plantas) - Faculdade de Ciências Agronômicas, Universidade Estadual Paulista, Botucatu, 2003.

MENDONÇA, C.G.; RAETANO, C.G.; MENDONÇA, C.G. Tensão superficial estática de soluções aquosas com óleos minerais e vegetais utilizados na agricultura. Engenharia Agrícola, Jaboticabal, v.27, p.16-23, 2007. Número Especial

MENDONÇA, C.G.; VELINI, E.D.; MARTINS, D.; MENDONÇA, C.G. Efeitos de surfatantes sobre a tensão superficial e a área de molhamento de soluções de glyphosate sobre folhas de tiririca. Planta Daninha, Viçosa - MG, v.13, n.3, p.355-365, 1999.

MOITA NETO, J.M. Molhamento e ângulo de contato. março de 2006. Teresina: Fundação de Amparo a Pesquisa do Estado do Piauí. Disponível em:

$<$ http://www.fapepi.pi.gov.br/ciencia/documentos/Molhamento.PDF>. Acesso em: 20 out. 2007. 
MONTÓRIO, G.A. Eficiência dos surfatantes de uso agrícola na redução da tensão superficial. 2001. 72 f. Tese (Doutorado em Agricultura) - Faculdade de Ciências Agronômicas, Universidade Estadual Paulista, Botucatu, 2001.

SILVA, F.M.L.; VELINI, E.D.; CORRÊA, T.M. Influência dos íons Mg, Ca, Fe, Cu e Zn sobre a tensão superficial estática de soluções contendo surfatante. Planta Daninha, Rio de Janeiro, v.24, n.3, p. 589-595, 2006.

SUN, J.; FOY, C.L. Phisico-chemical properties of several commercial organosilicones their blends, and selected other adjuvants. In: NALEWAJA, J.D.; GROSS, G.R.; TANN, R.S. (Ed.). Pesticide formulations and application systems. West Conshohocken: American Society for Testing and Materials, 1998. v.18, p.281-293. 